\title{
Early Detection of Defects through the Identification of Distortion Characteristics in Ultrasonic Responses
}

\author{
Pietro Burrascano*(D) and Matteo Ciuffetti \\ Dipartimento di Ingegneria, Università di Perugia, 06125 Perugia, Italy; matteo.ciuffetti@studenti.unipg.it \\ * Correspondence: pietro.burrascano@unipg.it
}

Citation: Burrascano, P.; Ciuffetti, M. Early Detection of Defects through the Identification of Distortion Characteristics in Ultrasonic

Responses. Mathematics 2021, 9, 850 . https: / / doi.org/10.3390/math9080850

Academic Editor: Mario Versaci

Received: 8 March 2021

Accepted: 12 April 2021

Published: 13 April 2021

Publisher's Note: MDPI stays neutral with regard to jurisdictional claims in published maps and institutional affiliations.

Copyright: (c) 2021 by the authors. Licensee MDPI, Basel, Switzerland. This article is an open access article distributed under the terms and conditions of the Creative Commons Attribution (CC BY) license (https:// creativecommons.org/licenses/by/ $4.0 /)$.

\begin{abstract}
Ultrasonic techniques are widely used for the detection of defects in solid structures. They are mainly based on estimating the impulse response of the system and most often refer to linear models. High-stress conditions of the structures may reveal non-linear aspects of their behavior caused by even small defects due to ageing or previous severe loading: consequently, models suitable to identify the existence of a non-linear input-output characteristic of the system allow to improve the sensitivity of the detection procedure, making it possible to observe the onset of fatigue-induced cracks and/or defects by highlighting the early stages of their formation. This paper starts from an analysis of the characteristics of a damage index that has proved effective for the early detection of defects based on their non-linear behavior: it is based on the Hammerstein model of the non-linear physical system. The availability of this mathematical model makes it possible to derive from it a number of different global parameters, all of which are suitable for highlighting the onset of defects in the structure under examination, but whose characteristics can be very different from each other. In this work, an original damage index based on the same Hammerstein model is proposed. We report the results of several experiments showing that our proposed damage index has a much higher sensitivity even for small defects. Moreover, extensive tests conducted in the presence of different levels of additive noise show that the new proposed estimator adds to this sensitivity feature a better estimation stability in the presence of additive noise.
\end{abstract}

Keywords: non-destructive evaluation; pulse compression; non-linear systems; Hammerstein model

\section{Introduction}

The use of techniques to detect non-linear behaviors in systems, aimed at early stage detection and identification of defects in structures and materials, is a rapidly evolving research field with great potential for application in industrial production and in periodic tests aimed at safety and durability. Increasingly sophisticated techniques are being consolidated for the early detection of imperfections, defects, and micro-fractures due to oxidation or resulting from the fatigue behavior of materials [1-3]. The usual ultrasonic techniques can detect flaws of the order of wavelength. To detect defects of smaller size using ultrasonic excitations of the same frequency, particular phenomena that induce non-linear behavior in the response must be considered, and this allows the detection of the much smaller flaws.

Non-destructive techniques for defect detection can be divided into linear and nonlinear. Taking ultrasonic techniques as a reference, linear methods are based on the estimation of parameters such as attenuation, transmission, and reflection coefficients, thus implicitly likening the analyzed system to a linear one whose impulse response is measured. Based directly on propagation phenomena, these techniques are capable of detecting, in the material, discontinuities of a size comparable to the wavelengths involved: this makes these techniques unsuitable for detecting extremely small fractures, if smaller than this size; increasing the frequency of the signal used may allow the detection of defects of proportionally smaller size, but is faced with limitations in the availability of suitable transducers 
and significant increases in propagation attenuation. The non-linear techniques have the potential to overcome, within certain limits, the wavelength constraint, as they aim at detecting how the waveform of the signal injected into the system changes due to the introduction of further harmonic components in the response of the non-linear system, which can make the signal waveform significantly different from that of the excitation signal.

The mechanism behind the formation of non-linear components in the response can be interpreted in a very simple way by considering the interaction between the crack facing surfaces when they are hit by the ultrasonic wave propagating through the medium: in the phase in which the propagating wave generates compression, a contact is created between the two faces of the crack, and this contact causes the propagation of the ultrasonic wave to occur in an unattenuated manner; in the semi-period in which the propagating wave, instead, generates tension in the medium, the two walls detach and the propagation occurs in a strongly different manner, such that the tension half-waves are significantly more attenuated. The mechanism that makes the behavior of the compression and tension phases different during each period is such as to produce a non-linear distortion of the propagating signal, which generates additional harmonic components in the response of the system, and this occurs even if the transverse dimensions of the crack are small compared to the wavelength of the propagating signal $[4,5]$.

A number of defect detection techniques based on non-linear behavior can be defined according to the particular aspect that is considered, among the features resulting from the non-linear characteristic of the examined system; one can distinguish between methods that detect the presence of higher order harmonics [6-8], methods that analyze the frequency shift of resonances [9,10], vibro-acoustic modulation methods [5,11], and frequency mixing methods, which highlight the production of non-linear combinations of harmonic components that have been generated due to different phenomena or propagation modes [12,13]. In the first case, the response is analyzed to detect the presence of higher order harmonics: e.g., in [6], it is shown how non-linearity due to the presence of damages manifests itself as sideband components in the spectrum of the received signal, while in [8], a technique based on pulse compression and the scalar subtraction method are compared under the same experimental conditions to highlight their respective sensitivities: a laboratory test experiment on mortar samples is performed to compare their ability to detect spectral components due to early damage in samples showing a non-linear response. To give a quantitative idea of the improvement that can be achieved in terms of resolution by considering non-linear phenomena, we report that in [8], the experimental results carried out on concrete bar adopting a swept sine signal, whose center frequency is $55 \mathrm{kHz}$, allowed the detection of an U-shaped notch in the middle of the bar, whose dimension in the direction of propagation is approximately $\lambda / 12$, i.e., allowing a range resolution at least 3 or 4 times higher than that of the usual ultrasound techniques. Nonlinear resonance acoustic spectroscopy studies nonlinear phenomena by analyzing the resonance modes of a material. Granular and micro-fractured materials always show a non-linear attenuation of the modulus of elasticity, even at very low strain levels. As a result, their resonance frequency shifts, harmonics are generated, and amplitude-dependent damping characteristics are observed. In undamaged materials, these phenomena are very weak. In damaged materials, they are considerably more pronounced. In [11], it is shown that non-fully bonded interfaces exhibit highly non-linear behavior: one of the consequences of such non-linear behavior is the modulation of a high-frequency ultrasonic wave by a low-frequency vibration. The vibration varies the contact area by modulating the phase and amplitude of the higher frequency test wave passing through the damaged interface. In [13], the authors consider the non-linear interaction of counter-propagating Lamb waves and the resulting resonance phenomenon, and analyze the resulting overall signal in the time domain in order to make a precise localization of the damage in the structure.

In our case, we will mainly refer to techniques that analyze higher order harmonics. These techniques have led to extremely interesting application results: for example, [14] explores the possible use of non-linear ultrasonic techniques, and in particular, the inter- 
modulation components, for detecting the cracking due to corrosion of steel reinforcements in concrete; in [15], a non-linear technique based on second-order harmonics is adopted to characterize the damage present in granite samples subjected to compressive loads, and it is shown that the non-linear parameter used is significantly more sensitive to the presence of damage than parameters based on linear techniques.

In the present paper, we show how a well-established technique for the identification of the non-linear Hammerstein model of the structure under investigation allows to define a new reliable damage indicator for an early detection of possible flaws, based directly on the parameters of the identified model. The validation of the procedure on a consolidated integral model of the physical structure allows to compare the reliability of the proposed method with that of a different damage indicator, proposed in the technical literature. The comparison is also performed in the presence of high levels of additive noise: even under these conditions, the innovative estimator we propose shows a higher sensitivity to small-sized defects and a higher stability in the estimated value.

The paper is organized as follows: in Section 2, the theoretical aspects of the procedure are described: Section 2.1 provides a description of the technique for the identification of non-linear systems based on the Hammerstein model. The identification technique adopted in this paper is based on (i) the use of a swept sine exponential signal as a pilot input to the nonlinear system and on (ii) the processing of the corresponding response by means of a pulse compression technique: the latter is described in Section 2.2; in the same Section 2.2, it is also highlighted how this technique is associated with an improvement of the signal-to-noise ratio (SNR) at the output of the matched filter. Section 2.3 then describes the damage indicators and the simulation system adopted for the subsequent experiments, and in particular, Section 2.3.1. describes the damage indicator proposed in the technical literature, and Section 2.3.2. defines the original harmonic index damage indicator that we propose in this paper. Section 2.3.3. describes the model adopted to generate the data considered to simulate the non-linear system that was adopted for the subsequent simulation experiments, an integral model known in the technical literature. Section 3 describes the experimental tests performed using the proposed new estimator, the results of which are compared with those of the other estimator considered, on synthetic experiments; the results obtained by using the two damage indicators are discussed and compared. In Section 4, some conclusions are drawn and the possible evolution of the research work is indicated.

\section{Modeling Non-Linearity for Damage Detection}

In principle, one of the simplest methods of assessing the non-linearity characteristics of a structure or material would be to measure the magnitude of the harmonics present in the system's response to a single sinusoidal excitation tone. This technique is difficult to apply in practice, except for systems operating in a frequency range close to DC. This is because the harmonic components quickly reach frequencies that are either outside the operating range of the system, or outside the measurable frequency range of the sensors used. This problem can be overcome by using different input signals, such as a couple of sinusoidal tones: this input produces, at the output of the non-linear system, harmonic components at frequencies which are linear combinations with integer coefficients of the tones at the input. Among these components, we can choose an intermodulation frequency close to the useful band of the system. The amplitude of this component allows to define other types of estimators of the degree of non-linearity in the behavior of the system. The amount of distortion, and therefore the amount of harmonic intermodulation components associated with the input signal, depends on the measuring modes and increases as the excitation signal level increases. For these reasons, the procedures for its measurement are codified in international standards [16].

These techniques, however, are based on the frequency analysis of the signal produced at the output of the system. They have undoubted advantages linked to their simplicity and are computationally efficient, but they involve all the limitations of spectral analysis, 
whether they refer to parametric methods based on the Fourier transform of the autocorrelation estimate (Blackman and Tukey) or are based on the direct application of the Fast Fourier Transform to the data (periodogram). These methods, due to the strong presence of sidelobes associated with them, imply the impossibility of detecting harmonic components of limited amplitude [17]. This can be a major disadvantage if we are interested in the early detection of the onset of non-linear effects and to the first occurrence of additional harmonic components related to such phenomena. The use of appropriate windows can only partially limit these effects.

Alternatives have been proposed to the frequency methods, as for instance, those based on a comparison of the input and output signals in the time domain, as is done for example with the scaling subtraction method (SSM) technique: it extracts the non-linear characteristic of the output waveform relying on the breaking of the superposition principle that is induced by the elastic non-linearity of the system considered $[7,8]$.

In our case, we focus on methods based on spectral analysis, so we can increase their sensitivity through the use of physical system modeling techniques. In fact, one often has more knowledge of the process generating the data than is available from the data alone, or at least is able to make reasonable assumptions about the nature of the system generating the data. The use of a priori information or assumptions makes it possible to define a model that is a good approximation of the generating process [17]. This is what is done, using the Hammerstein model of the non-linear system.

\subsection{Identification of the Hammerstein Model of Non-Linear Systems}

Phenomenological models are widely used to characterize the non-linear link between input and output in a non-linear system: this approach to the study of non-linear systems was first proposed by Volterra, who defined a functional representation that gives an explicit input/output relationship when both input and output are bounded [18,19].

However, the Volterra series consists of an infinite number of terms and therefore in practical applications its truncation is necessary. Furthermore, even if the order of the Volterra series is truncated, the number of coefficients required to define the model quickly becomes very large as the degree of non-linearity increases.

Consequently, several techniques have been developed that can be interpreted as simplified versions of those proposed by Volterra. Among them, the generalized Hammerstein model of order $N_{H}$ has been defined: the corresponding structure consists of $N_{H}$ parallel branches, each of which includes a static non-linearity followed by a linear filter, as shown in Figure 1. Although less general than the Volterra series expansion, the Hammerstein model shows the ability to define accurate models even in the case of strong non-linearities, while using a reduced number of parameters [20]. Furthermore, a very efficient method for the identification of the Hammerstein model by using swept-sine signals has been defined in the literature [21], based on a Pulse Compression (PuC) technique, i.e., the use of a coded signal as excitation and a corresponding matched filter.

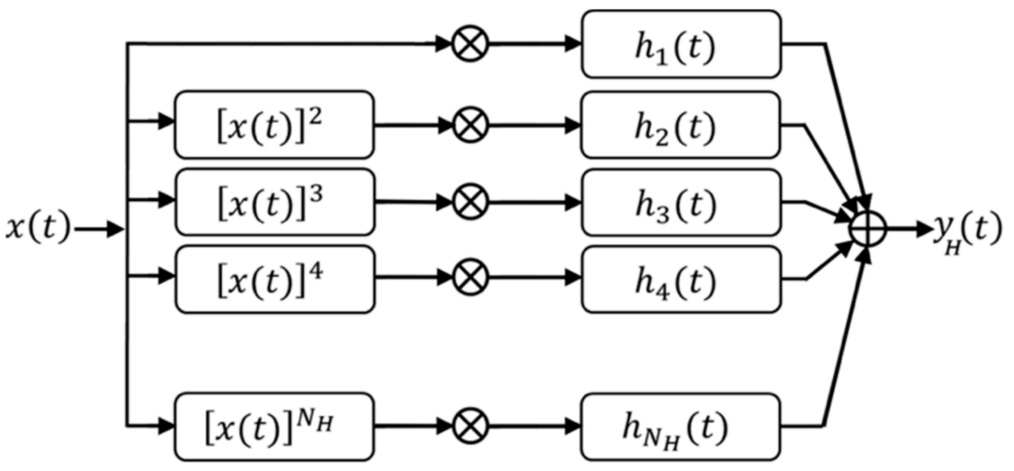

Figure 1. The Hammerstein models. 
We briefly summarize the Hammerstein model identification procedure based on the PuC technique. Let us assume that the non-linear system can be represented by its Hammerstein model of order $N_{H}$, and that the input signal is of the harmonic type: $x(t)=\cos (\phi(t))$, whose angular frequency $\omega(t)=\frac{d \phi(t)}{d t}$ varies in time. In this case, the following relation holds between the vector $[\cos (k \phi(t))]$ of harmonics of the input signal, up to the $N_{H}-t h$, order, and the vector $\left[\cos (\phi(t))^{k}\right]$ of its powers up to the $N_{H}-t h$ :

$$
[\cos (k \phi(t))]=\left[A_{c}\right]\left[\cos (\phi(t))^{k}\right] ; 1 \leq k \leq N_{H}
$$

in which $\left[A_{c}\right]$ is the matrix of the coefficients of the Chebyshev polynomials of the first kind. Using the inverse of (1), we can express the output $y_{H}(t)$ of the model as:

$$
\begin{aligned}
y_{H}(t) & =\left[\cos (\phi(t))^{k}\right]^{T} \otimes[h(t)]=\left[\left[A_{c}\right]^{-1} \cos (k \phi(t))\right]^{T} \otimes[h(t)] \\
& =[\cos (k \phi(t))]^{T} \otimes\left[\left[A_{c}\right]^{-1}\right]^{T}[h(t)]=[\cos (k \phi(t))]^{T} \otimes[g(t)],
\end{aligned}
$$

where $\otimes$ indicates convolution and the vector $[h(t)]$ contains the ordered sequence of the $N_{H}$ impulse responses which characterize the branches of the Hammerstein model and the functions $g_{k}(t)$ in the vector $[g(t)]=\left[\left[A_{c}\right]^{-1}\right]^{T}[h(t)]$ are linear combinations of the $\mathrm{h}_{k}(\mathrm{t})$ in the vector $[h(t)]$.

The identification technique of the Hammerstein model is based on the above formulation: let us consider a signal of the harmonic type whose angular frequency increases in time according to an exponential law; in this case, the frequencies associated with two different time instants, namely $t_{1}$ and $t_{2}=t_{1}+\Delta t$, will be in a fixed ratio, whatever $t_{1}$ is, and the value of their ratio will depend only on $\Delta t$. In particular, we can choose $\Delta t=\Delta t_{k}$ in such a way that the corresponding frequency ratio is equal to an integer $\mathrm{k}$, i.e., it is in the ratio of harmonics. Consider that such a signal $x(t)$ is input to the non-linear system and that we define a filter $\psi(t)$ matched to the excitation signal, i.e., such that when the signal $x(t)$ is input to $\psi(t)$ the response of the filter is the Dirac delta function $\delta(t)$. If we filter the output $y_{H}(t)$ of the Hammerstein model through $\psi(t)$, the output $\mathrm{u}(t)$ of the matched filter will be:

$u(t)=y_{H}(t) \otimes \psi(t)=\left[\cos [k \phi(t)]_{c}\right]^{T} \otimes[g(t)] \otimes \psi(t)=\left\{\left[\hat{\delta}\left(t+\Delta t_{k}\right)\right]^{T}\right\} \otimes[g(t)]$,

where:

- $\quad$ each element $\hat{\delta}\left(t+\Delta t_{k}\right)$ is an approximate version of the Dirac delta function, delayed by the quantity $\Delta t_{k}=L \ln (k)$ associated with the k-th harmonic of the signal;

- $\quad L=\ln \left(f_{2} / f_{1}\right) / T$ defines the time variation rapidity of the instantaneous frequency of the exponential swept sine signal, which sweeps between the frequencies $f_{1}$ and $f_{2}$ in a time $T$;

The functions $g_{k}(t)$ can be obtained by extracting appropriate time sections from the output signal $u(t)$; using relations in accordance with (2), it is easy to obtain the functions $h_{k}(t)$ that characterize the Hammerstein model from the $g_{k}(t)$ obtained in this way, evaluating them through a simple linear transformation whose coefficients are directly derived from those of the Chebyshev polynomials of the first kind [21,22]. Namely, we can write:

$$
[h(t)]=\left[A_{c}\right]^{T}[g(t)] .
$$

This identification technique turns out to be particularly suitable for the early detection we are interested in, as it allows us to adjust the signal-to-noise ratio through the most opportune choice of the parameters that characterize the coded signal used for the excitation of the system. Figure 2 provides a schematic description of the PuC procedure for identifying the Hammerstein model of the non-linear system under consideration: the functions $\mathrm{g}_{k}(\mathrm{t})$ highlighted by red boxes at the output of the matched filter are those whose transformation through the matrix $\left[A_{c}\right]$ allows us to obtain the kernels $\mathrm{h}_{k}(\mathrm{t})$ present in the 
Hammerstein model in Figure 1. The functions $g_{k}(t)$ are also those that will be used in the definition of the indicator that we propose in a later section of the paper.

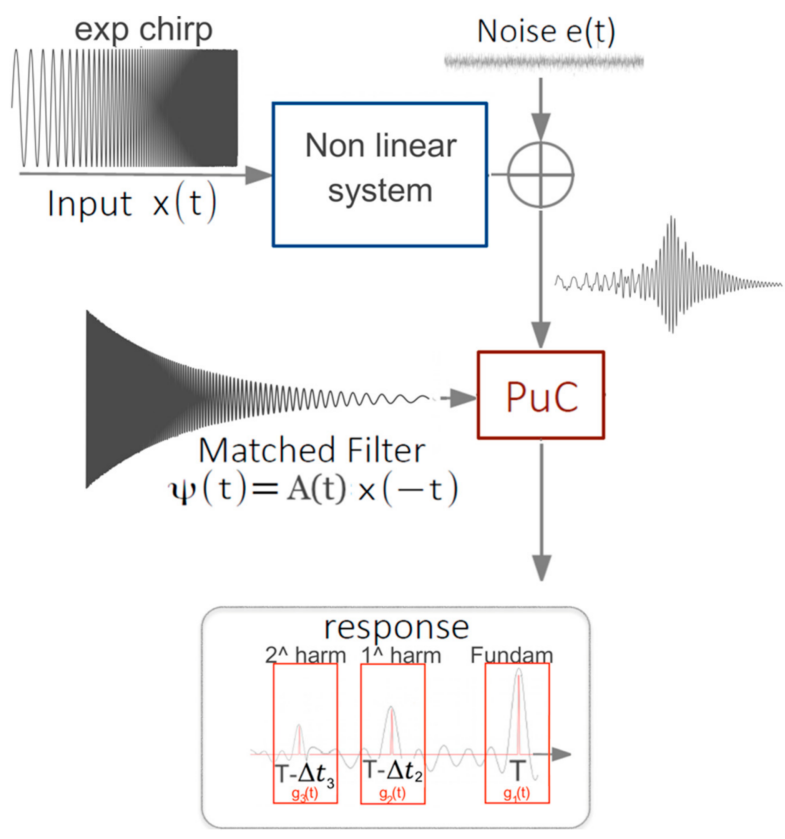

Figure 2. The Pulse Compression $(\mathrm{PuC})$ procedure for identifying the Hammerstein model.

\subsection{Pulse Compression and Signal-to-Noise Ratio}

Among the various applications of coded sequences $[23,24]$, pulse compression, which originated in the field of radar techniques [25], has recently found numerous important areas of application [26-28]. In the case we are considering, of using the PuC technique for the identification of the Hammerstein model, swept sine signals of exponential type are used, and the signal-to-noise ratio can be adjusted by choosing the most opportune duration for this excitation signal.

In fact, one of the ways of considering $\mathrm{PuC}$ is to consider the effect of increasing signalto-noise ratio (SNR) that it allows to obtain in a specific time interval: the filter matched to the input signal operates only on the phases of its harmonic components, and it does not alter their amplitudes. In this way, it compresses the signal duration in the time domain, but does not alter its energy content, as it only modifies its group delay. Consequently, if we denote by $\mathrm{P}$ and $\mathrm{P}^{\prime}$ the average powers and by $\mathrm{T}$ and $\mathrm{T}^{\prime}$ the signal durations before and after compression, we will then have $\mathrm{P} \times \mathrm{T}=\mathrm{P}^{\prime} \times \mathrm{T}^{\prime}$, from which the average signal power after compression can be written as $\mathrm{P}^{\prime}=\mathrm{P} \times\left(\mathrm{T} / \mathrm{T}^{\prime}\right)$. This way of expressing the average power shows that the ratio $\left(\mathrm{T} / \mathrm{T}^{\prime}\right)$ can be seen as an equivalent power gain. For impulsive signals, such as that occurring after compression, the duration of the signal is inversely proportional to their bandwidth: $\mathrm{B} \propto 1 / \mathrm{T}$; consequently, in many cases the equivalent power gain is expressed as TB: if we have an amplifier capable of providing a power of $\mathrm{P}$ [W], after compression in the interval of duration $\mathrm{T}^{\prime}$ of the compressed signal, we have a signal equivalent to what we would have had with an amplifier TB times more powerful. If the noise has the same power, TB therefore translates into a gain in SNR.

\subsection{Innovative Indices for Early Damage Detection}

Having available accurate modeling techniques makes it possible to define highly sensitive indicators for the early detection of damage to the structure, based on the nonlinear nature of the response of the system under consideration. We will examine an indicator, proposed in [22], that has demonstrated excellent characteristics, analyze its behavior and propose an original indicator. 


\subsubsection{The $D I_{1}$ : Ratio of the Non-Linear Energy to the Linear Energy}

The $D I_{1}$ damage indicator is based on a brilliant method to separate the linear contribution from the non-linear one. The method is based on the Hammerstein model of the physical structure: having identified the Hammerstein model of the non-linear system allows to extract from it an indicator of the degree of non-linearity of the system by separating the contribution to the output signal due to the branches of the model representing the non-linear components of the system, and comparing this contribution to the one related to the branch representing the linear part. In [22], the authors make use of this model to define, among others, the indicator:

$$
D I_{1}=\frac{\int_{f_{1}}^{f_{2}}\left|S^{N L}(f)\right|^{2} d f}{\int_{f_{1}}^{f_{2}}\left|S^{L}(f)\right|^{2} d f},
$$

where $S^{L}(f)$ and $S^{N L}(f)$ are, respectively, the Fourier transforms of the response of the linear branch and the sum of the responses of the non-linear branches of the Hammerstein model, once identified, when the exponential sine sweep signal is input; $f_{1}$ and $f_{2}$ are the extreme frequencies of the exponential sine sweep signal that is used as input to the system in the phase of identification of the non-linear system, the same signal used as input to the Hammerstein model, once identified, to estimate the damage index $D I_{1}$.

\subsubsection{The Proposed Harmonic Index}

The definition of an estimator for the degree of non-linearity can only be based on the separation of the linear and non-linear parts. The good results produced by the damage index $D I_{1}$ show that, in this field too, the use of a priori information, in the form of a model of the structure to be represented, significantly improves the estimation sensitivity.

The aspect that we have considered to focus on in examining the performances of the estimator, is the degree of immunity to noise of the data collected: the conditions in which the acquisition of the measurement data take place are in fact very frequently limited in power, thus noise level overrides small signal variations and this makes it difficult to detect small differences between the responses of an intact specimen and a faulty one of the system under examination, in case we are interested in an early manifestation of a possible defect.

The $D I_{1}$ technique implies that once the Hammerstein model is identified, it is used by injecting the noise-affected swept sine signal at its input: the sum of signal and noise is processed by each of the parallel branches in the model. The sum of the energies of the output signals from the non-linear branches is divided by the energy of the output signal from the linear branch: this ratio is the $D I_{1}$ index. The effect of the input noise, altered by the processing phases in the different branches of the model, directly reflects on the estimated value for the $D I_{1}$ index. This estimator therefore does not make use of the improvements in the SNR ratio that can be achieved through the PuC technique, even though the $\mathrm{PuC}$ technique was used in the previous Hammerstein model identification phase.

As far as the damage index is concerned, our proposal is therefore to define the indicator using quantities calculated in the PuC phase, a procedure that is in any case implemented for the identification of the Hammerstein model: following this approach has the advantage of using the PuC procedure for two purposes, and furthermore of having a defect index estimated under more advantageous equivalent SNR conditions.

There is a second argument in support of the potential offered by a damage index definition based on quantities calculated in the PuC phase, thus considering the signal $\mathrm{u}(t)$ at the output of the matched filter: the estimator proposed in [22] makes use of the Hammerstein model once identified, and measures the energy of the linear and non-linear parts of the output signal of the model when an exponential sine sweep signal feeds into the parallel branch structure: this input signal has, by definition, a spectral energy distribution strongly unbalanced towards one of the extremes of the covered band: in the case of a signal with exponentially increasing frequency (up-chirp), the harmonic content 
is mainly concentrated towards the lowest range of frequencies. Assuming that the noise superimposed in the measurement phase is assimilable to a Gaussian white noise, it is evident how the ratio between useful signal and noise is strongly unequal in the different frequency ranges. This fact makes the estimator proposed in [22] particularly exposed to the effects of noise in the frequency ranges where the signal-to-noise ratio is less advantageous, frequency bands that might be particularly important for detecting small defects, which are important for early detection: it is therefore particularly important to check the sensitivity and robustness of the estimator in the presence of noise. In order to verify these aspects, we report in the next paragraph the results of some simulations carried out in a wide range of signal-to-noise ratios, evaluation including also very low SNR values.

The proposal of a new estimator starts from the above considerations and, being always based on the Hammerstein model, does not use the energy of the signal at the model output, separated between linear and non-linear components, but directly the energy associated to the impulsive functions $\mathrm{g}_{k}(\mathrm{t})$ in the vector $[g(t)]$ which are estimated during the model identification phase, as reported in equation (3). These functions directly carry information about the different harmonics that are generated in the response of the non-linear system; moreover, their estimation, carried out downstream of the matched filter, compensates in this way the non-uniformity in the energy content of the excitation signal, which is automatically equalized by the matched filter.

Based on this consideration, we propose the following harmonic index of damages in the structure:

$$
H I=\frac{\sum_{k=2}^{N_{H}} \int\left(\mathrm{g}_{k}(\mathrm{t})\right)^{2} d t}{\int\left(\mathrm{g}_{1}(\mathrm{t})\right)^{2} d t},
$$

where the integrals are carried out in the time domain, extended to the entire duration of each $\mathrm{g}_{k}(\mathrm{t})$ function.

\subsubsection{The Simulated System}

The experiments were performed by generating synthetic signals using the same model used in [22], a model previously proposed and used in [29]. The system that has been chosen is a one degree of freedom system, of the spring-mass-damper (SMD) type as shown in Figure 3. The damage in these systems has been introduced through a bi-linear stiffness $k[x(t)]$, and allows to simulate in a very simple way a breathing fracture [29]. For these fractures there is a lower stiffness when the fracture is open than when it is closed. Consequently, the bi-linear stiffness is defined as follows:

$$
k[x(t)]=\left\{\begin{array}{c}
\mathrm{k}^{I} \\
(1-\alpha) \mathrm{k}^{I}
\end{array} .\right.
$$

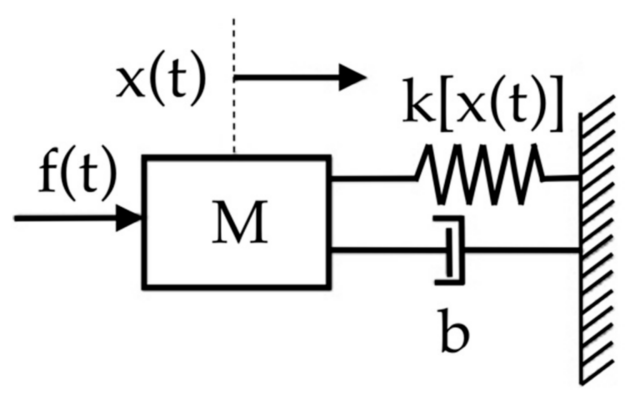

Figure 3. The one degree of freedom system, of the spring-mass-damper.

In this definition, $\mathrm{k}^{I}$ indicates the linear stiffness of the original undamaged system and the damage parameter of the simulation model is the coefficient $\alpha$. If $\alpha=0$, the stiffness is fully linear and the system is intact. If $\alpha=1$, the stiffness is zero when the fracture is open, and consequently the system is fully damaged. 
We have chosen a single-input, single-response (SISO) system whose input is the force $f(t)$ applied to the mass $M$; the output is the displacement $x(t)$ of the mass $M$, as shown in Figure 3.

The effect of noise, in this case, may affect the quality of the estimation of the $g_{k}(t)$ functions if the output signal used in the identification phase is affected by additive noise.

\section{Experimental Results}

In accordance with [22], we generated data from the system defined by relation (7) using the following parameters: $M=1[\mathrm{~kg}], B=2[\mathrm{~N} \mathrm{~s} / \mathrm{m}]$, and $\mathrm{k}^{I}=20000[\mathrm{~N} / \mathrm{m}]$.

An input signal with the characteristics of an exponential sine sweep with increasing frequency was used to identify the Hammerstein model and subsequently to estimate the value of the damage indicators under comparison. The resonance frequency of the mechanical system described above is $f_{R}=22.5[\mathrm{~Hz}]$ for the undamaged system. Consequently, according to [22], we selected the initial frequency $f_{1}=2.25[\mathrm{~Hz}]$ and final frequency $f_{2}=225[\mathrm{~Hz}]$. The duration chosen for the excitation signal was $\mathrm{T}=9.36[\mathrm{~s}]$.

The response of this system was simulated within the Mathematica ${ }^{(r)}$ environment. A zero mean, white Gaussian noise was added to the input of the simulator to consider the environmental noise. In order to allow a complete comparison with the data reported in the paper [22], we assumed that the measurement system does not add any perceptible noise component to the signal detected. The variance of the noise was chosen according to the desired signal-to-noise ratio: initially the results for SNR of 60 and $30 \mathrm{~dB}$ are reported, as in [22]. Measurements with much higher noise levels were also carried out, such that the SNR ratio was brought down to a value of $0 \mathrm{~dB}$. Each simulation was performed for the same range of $\alpha$ damage parameter: the values considered were between $\alpha=0 \%$ and $\alpha=50 \%$ for all simulations. For each value of $\alpha$ and for each value of the signal-to-noise ratio, the simulation was repeated 30 times in order to calculate the mean value and the standard deviation of each one of the damage estimators under comparison in the presence of noise.

Figure 4 shows, in the four panels, the trend in the case of SNR $=60 \mathrm{~dB}$ and in the case of SNR $=30 \mathrm{~dB}$ of the mean value and the standard deviation $( \pm 2 \sigma)$ of the estimator of the two damage indicators being compared. The abscissa shows the extent of damage $\alpha$, whose value varies between $0 \%$ and $50 \%$. The mean value and the standard deviation are calculated, as in all subsequent cases, on 30 independent tests.

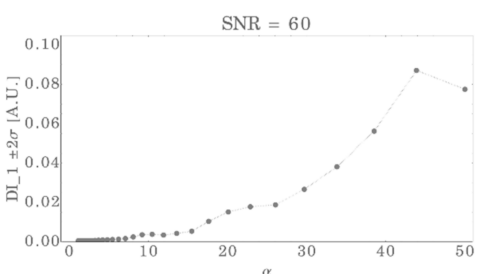

(a)

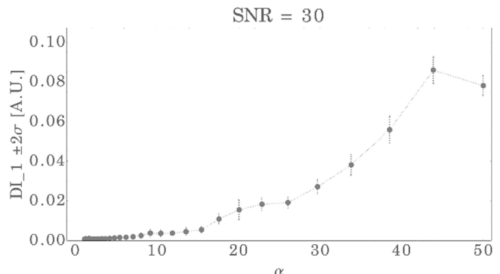

(c)

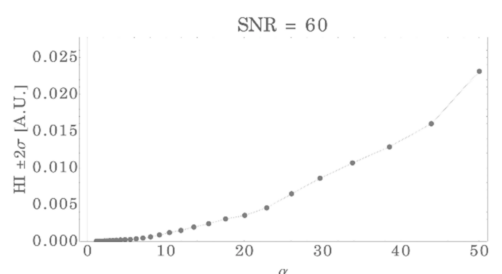

(b)

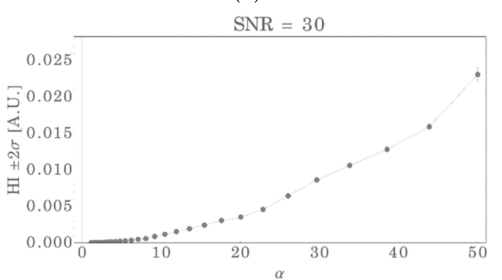

(d)

Figure 4. Mean value and standard deviation $( \pm 2 \sigma)$ of the estimators depending on the amount of damage $\alpha$. (a) Damage indicator $D I_{1} @ \mathrm{SNR}=60 \mathrm{~dB}$; (b) The proposed damage indicator $\mathrm{HI} @$ $\mathrm{SNR}=60 \mathrm{~dB} ;(\mathbf{c})$ damage indicator $D I_{1} @ \mathrm{SNR}=30 \mathrm{~dB} ;(\mathbf{d})$ The proposed damage indicator $\mathrm{HI} @$ $\mathrm{SNR}=30 \mathrm{~dB}$. 
At such high levels of the signal-to-noise ratio, and with this type of representation, the trend of the mean values of each damage indicator in the case of 30 and $60 \mathrm{~dB}$ is actually indistinguishable: both indicators at these noise levels provide very stable indications. Only a greater variability around the mean value in the case of noise at $30 \mathrm{~dB}$ for $D I_{1}$ can be seen.

In addition to highlighting the stability of both estimators, Figure 4 shows some interesting aspects for comparing the two damage indicators: (1) the greater stability of the estimate obtained with the $\mathrm{HI}$ indicator that we propose compared to that with the $D I_{1}$ indicator, as can be seen from the significantly lower $\sigma$ values in the first case; (2) the more gradual decrease in the value of the estimator as the magnitude of the damage index $\alpha$ decreases. This latter aspect is particularly interesting if we want useful indications for early detection of damage, i.e., for low values of $\alpha$. To analyze this aspect in more detail, it may be useful to report the same values of Figure 4, panels (c) and (d), in a double logarithmic scale, in order to better evaluate the regularity of the variation of the trend of the indicators as $\alpha$ varies, especially in the lower range. In panels (a) and (b) of Figure 5, the trends of the two indicators in the case of SNR $=30 \mathrm{~dB}$ are plotted on a double logarithmic scale: their comparison shows a flattening of the curve of the $D I_{1}$ as $\alpha$ decreases, while the $\mathrm{HI}$ indicator decreases in a regular way up to the minimum $\alpha$ values. The HI therefore shows greater sensitivity than the $D I_{1}$ to even minimal damage, at the same noise level.

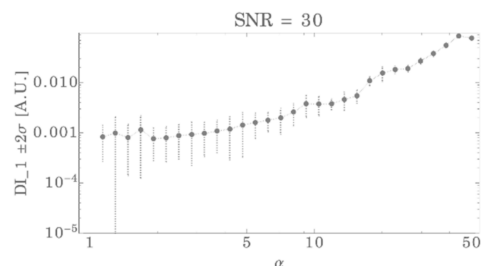

(a)

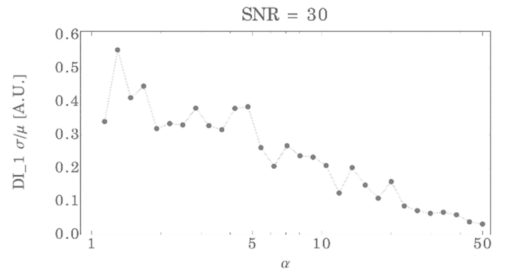

(c)

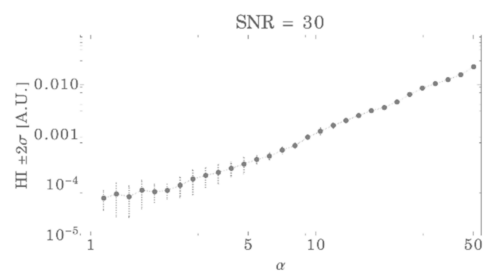

(b)

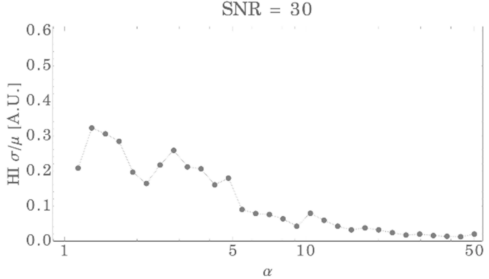

(d)

Figure 5. Mean value and standard deviation $( \pm 2 \sigma)$ of the estimators depending on the amount of damage $\alpha$. (a) Double logarithmic scale representation of damage indicator $D I_{1} @ \mathrm{SNR}=30 \mathrm{~dB}$; (b) double logarithmic scale representation of the proposed damage indicator $\mathrm{HI} @ \mathrm{SNR}=30 \mathrm{~dB}$; (c) $\left(\frac{\sigma}{\mu}\right)$ for $D I_{1} @ \mathrm{SNR}=30 \mathrm{~dB}$; (d) $\left(\frac{\sigma}{\mu}\right)$ for $\mathrm{HI} @ \mathrm{SNR}=30 \mathrm{~dB}$.

Panels (c) and (d) of the same Figure 5 show a comparison of the trends of the ratio $\left(\frac{\sigma}{\mu}\right)$ between the estimated standard deviation $\sigma$ and average value $\mu$, plotted as the entity $\alpha$ of the damage varies: the comparison between the two curves shows for both a value of the ratio that decreases as the extent of the damage $\alpha$ increases: as expected, a more evident damage gives rise to more stable estimates. The values of the ratio are however very different between the two curves, confirming the greater stability of the estimate of the $\mathrm{HI}$ indicator in the presence of noise.

We decided to further investigate the comparison between the two damage indicators in relation to their sensitivity to small damage, and their ability to provide a reliable estimated value even in the presence of high noise levels. To this end, we have carried out further tests in conditions of increasing noise level, down to $\mathrm{SNR}=0 \mathrm{~dB}$. The results are reported in Figures 6-8, relating respectively to SNR values of 10, 5, and $0 \mathrm{~dB}$. The three Figures 6-8 are organized in the same way as Figure 5: in each, the panels (a) and (b) report, for the two indicators considered, the average value and the standard deviation, estimated 
on 30 repetitions, as a function the values of the entity of the damage $\alpha$. In each figure, panels (c) and (d) show, for the two indicators considered, the trend of the $\left(\frac{\sigma}{\mu}\right)$ ratio as the extent of the damage parameter $\alpha$ varies. As with all other tests, each $\sigma$ and $\mu$ value is estimated on 30 independent tests.

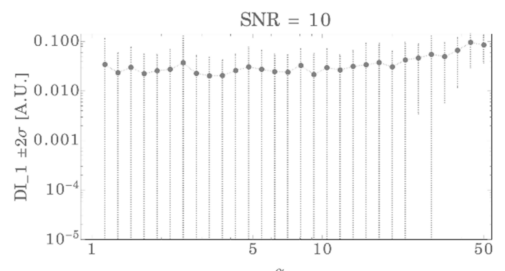

(a)

$\mathrm{SNR}=10$

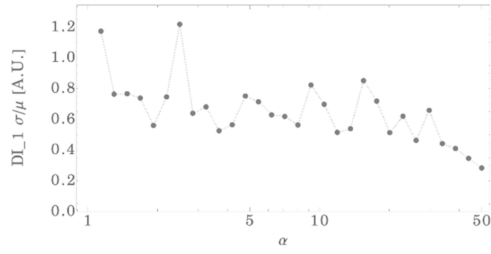

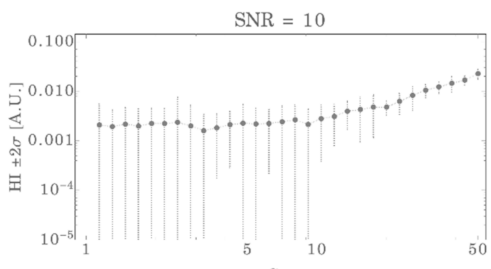

(b)

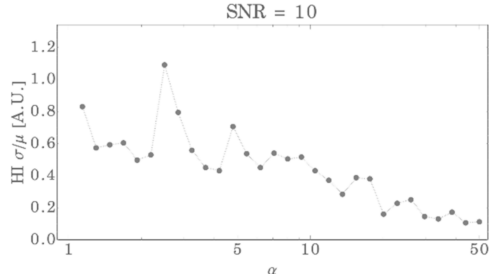

Figure 6. Mean value and standard deviation $( \pm 2 \sigma)$ of the estimators depending on the amount of damage $\alpha$. (a) Double logarithmic scale representation of damage indicator $D I_{1} @ S N R=10 \mathrm{~dB}$; (b) double logarithmic scale representation of the proposed damage indicator $\mathrm{HI} @ \mathrm{SNR}=10 \mathrm{~dB}$; (c) $\left(\frac{\sigma}{\mu}\right)$ for $D I_{1} @ \mathrm{SNR}=10 \mathrm{~dB} ;(\mathbf{d})\left(\frac{\sigma}{\mu}\right)$ for $\mathrm{HI} @ \mathrm{SNR}=10 \mathrm{~dB}$.

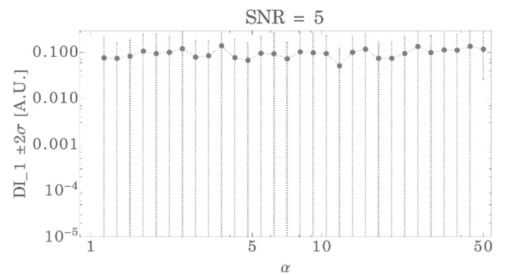

(a)

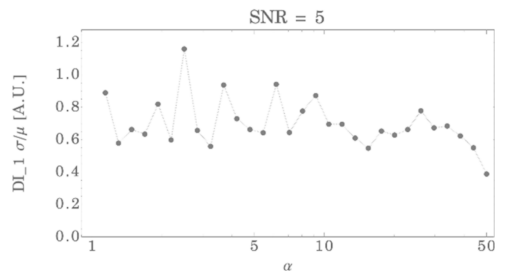

(c)

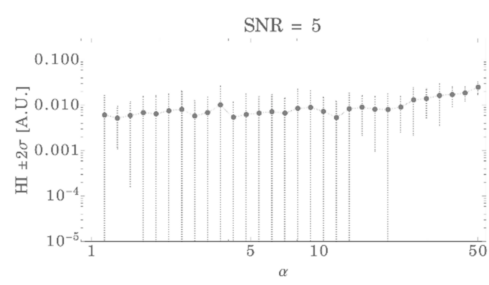

(b)

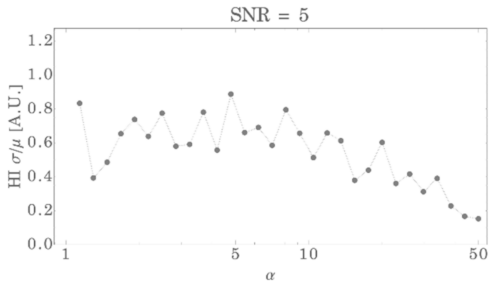

(d)

Figure 7. Mean value and standard deviation $( \pm 2 \sigma)$ of the estimators depending on the amount of damage $\alpha$. (a) Double logarithmic scale representation of damage indicator $D I_{1} @ \mathrm{SNR}=5 \mathrm{~dB}$; (b) double logarithmic scale representation of the proposed damage indicator $\mathrm{HI} @ \mathrm{SNR}=5 \mathrm{~dB}$; (c) $\left(\frac{\sigma}{\mu}\right)$ for $D I_{1} @ \mathrm{SNR}=5 \mathrm{~dB}$; (d) $\left(\frac{\sigma}{\mu}\right)$ for $\mathrm{HI} @ \mathrm{SNR}=5 \mathrm{~dB}$.

The figures clearly show how, as the noise level increases, the quality of the estimate for both damage indicators worsens. In particular for SNR equal to $0 \mathrm{~dB}$, both demonstrate that they are unable to modify their average value as a function of the parameter $\alpha$. For SNR equal to $5 \mathrm{~dB}$, the $\mathrm{HI}$ indicator we propose shows some, albeit limited, ability to detect the presence of damage exceeding $\alpha=10 \%$, while the $D I_{1}$ shows no variation. These different behaviors of the two damage indicators appears more evident for $\mathrm{SNR}=10 \mathrm{~dB}$. 


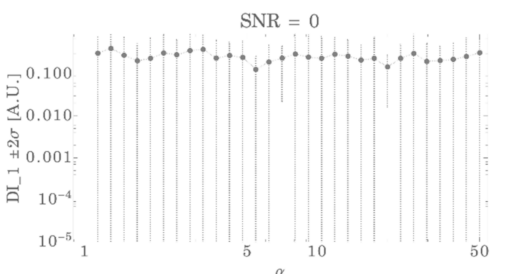

(a)

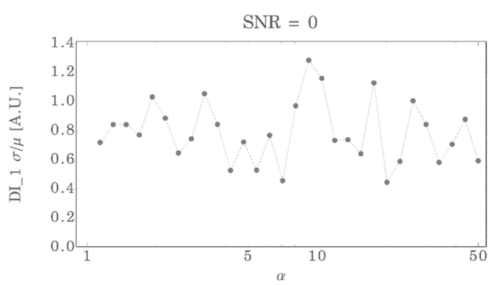

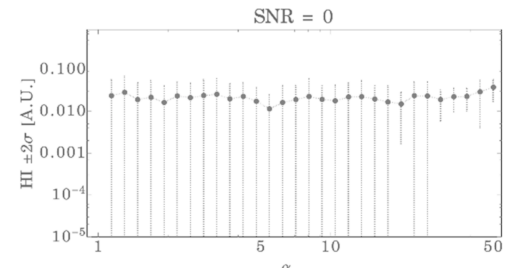

(b)

$\mathrm{SNR}=0$

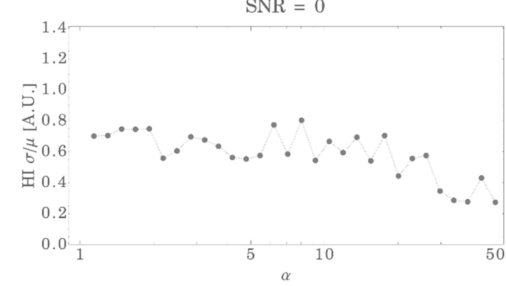

(d)

Figure 8. Mean value and standard deviation $( \pm 2 \sigma)$ of the estimators depending on the amount of damage $\alpha$. (a) Double logarithmic scale representation of damage indicator $D I_{1} @ \mathrm{SNR}=0 \mathrm{~dB}$; (b) double logarithmic scale representation of the proposed damage indicator $\mathrm{HI} @ \mathrm{SNR}=0 \mathrm{~dB}$; (c) $\left(\frac{\sigma}{\mu}\right)$ for $D I_{1} @ \mathrm{SNR}=0 \mathrm{~dB}$; (d) $\left(\frac{\sigma}{\mu}\right)$ for $\mathrm{HI} @ \mathrm{SNR}=0 \mathrm{~dB}$.

It is also interesting to observe that the $\sigma$ value significantly decreases starting from the $\alpha$ damage values for which its average value $\mu$ begins to change, showing that the defect can be detected by the damage index considered. In all these cases the $\left(\frac{\sigma}{\mu}\right)$ ratio relating to the $\mathrm{HI}$ indicator that we propose is lower than that relating to the $D I_{1}$ indicator, thus showing that, even in conditions of high noise level, the damage $\mathrm{HI}$ indicator tends in any case to be more reliable.

A fundamental aspect which emerges from a comparative analysis of the different figures describing our experiments is that, in the case of a very low SNR, and especially for small alterations of this signal due to defects (low $\alpha$ values), neither of the two methods is able to detect the presence of defects (see for example the curves for SNR $=0 \mathrm{~dB}$ : the value of both indices does not change when the entity of the defect, i.e., the value of $\alpha$, varies).

On the other hand, when the SNR is extremely high, especially for high $\alpha$ values, the presence of the defect is so evident that both indicators detect it without problems (see for example the curves for $\mathrm{SNR}=60$ or $30 \mathrm{~dB}$ : the value of both indices grows with regularity as $\alpha$ increases, i.e., as the extent of the defect raises).

The comparison between the two indicators is therefore played in verifying which method is able to detect first (i.e., for a lower $\alpha$ value, SNR being equal) the presence of defects. We can verify this, for each SNR, by increasing the $\alpha$ value. For very low $\alpha$ values, none of the indicators does change its value as the size of the defect increases: in fact, the noise is such, compared to the variations due to the defect, that the estimator does not change its value, that is, it does not detect the defect. The $\alpha$ value by which the estimator value begins to increase, evidences the beginning of the sensitivity zone for that indicator. So, it is not so much the absolute value of the estimator to be relevant, as it is going to verify for which $\alpha$ value the index begins to modify its value.

For example, the curves for SNR $=5 \mathrm{~dB}$ show a substantially constant $D I_{1}$ for the whole range, up to $\alpha=50 \%$, while the $\mathrm{HI}$ estimator begins to increase its value regularly starting from $\alpha=13 \%$; further confirmation of this behavior is highlighted by the significant decrease in the variability of the estimates around their average value starting from the same values of the $\alpha$ parameter (13\%). We have decided to further highlight these aspects, which we consider interesting for the comparison, by reporting-for each SNR — the trends of the $\left(\frac{\sigma}{\mu}\right)$ ratio as the $\alpha$ varies. Consistently with the observations just made, the trend of this curve confirms that the $\mathrm{HI}$ indicator shows that it detects the presence of defects even 
in situations in which the $D I_{1}$ is still made insensitive by the environmental noise present in the data.

Similar behaviors are found in the case of the curves for $\mathrm{SNR}=10 \mathrm{~dB}$, for which the indicator $D I_{1}$ enters the sensitivity zone for parameter $\alpha=30 \%$, while the $\mathrm{HI}$ indicator we propose is sensitive to variations in the extent of the defect already for $\alpha=10 \%$.

\section{Conclusions}

The non-linear techniques appear as a frontier for an improvement of the defect detention capacity at the early stage of defect formation. We have taken as a reference the $D I_{1}$, a technique that has proved to be effective and we have analyzed it thoroughly in order to improve its performance in the ability to provide reliable indications even in the presence of minor defects and in the presence of significant noise levels during data acquisition.

We have verified the possibility of using parameters that the $D I_{1}$ technique already considered in a preliminary phase, and we have used them to define the harmonic index $\mathrm{HI}$, a novel damage indicator. The experimental tests carried out by repeating the same experiment reported in the technical literature, in addition to finding the same values for the reference index $D I_{1}$, made it possible to find that the hypotheses of greater robustness to noise of the new proposed $\mathrm{HI}$ indicator are verified.

An accurate analysis of the trend of the two indicators for low damage values also highlighted a greater sensitivity of the new $\mathrm{HI}$ indicator for small damage, an aspect that is particularly important if one is interested in early detection of defects. Finally, we carried out numerous tests with remarkably high noise levels, to compare the robustness level of the two damage indicators and to obtain indications of the operating limits of both damage indicators. In addition, the new $\mathrm{HI}$ indicator we proposed has shown to have greater sensitivity and to provide indications less affected by the measurement noise.

The aim of the present work was to report the results of evaluations comparing the innovative indicator we defined with an indicator of very good performance that was already available in the technical literature. The comparisons had to be made under the same operating conditions, and this was done accurately using data obtained through simulations.

The extremely comforting results in terms of the stability of the estimated value as the extent $\alpha$ of the damage varies, and the robustness with respect to measurement noise, push this research activity towards carrying out a verification of the technique on experimentally measured data: this will allow a further comparison with other techniques of early damage detection.

Author Contributions: Conceptualization, P.B. and M.C.; Formal analysis, P.B. and M.C.; Software, P.B. and M.C.; Writing-original draft, P.B. and M.C. Both authors contributed substantially to the present work and have read and agreed to the published version of the manuscript.

Funding: This research received no external funding.

Institutional Review Board Statement: Not applicable.

Informed Consent Statement: Not applicable.

Data Availability Statement: Experiments carried out with data generated by the described synthetic model.

Acknowledgments: The authors thank the editor of this Special Issue for the encouragement and patient support.

Conflicts of Interest: The authors declare no conflict of interest.

\section{References}

1. Tsyfansky, S.; Beresnevich, V. Detection of fatigue cracks in flexible geometrically non-linear bars by vibration monitoring. J. Sound Vib. 1998, 213, 159-168. [CrossRef]

2. Bayma, R.S.; Zhu, Y.; Lang, Z.Q. The analysis of non-linear systems in the frequency domain using non-linear output frequency response functions. Automatica 2018, 94, 452-457. [CrossRef] 
3. Su, Z.; Zhou, C.; Hong, M.; Cheng, L.; Wang, Q.; Qing, X. Acousto-ultrasonics-based fatigue damage characterization: Linear versus non-linear signal features. Mech. Syst. Signal Process. 2014, 45, 225-239. [CrossRef]

4. Broda, D.; Staszewski, W.J.; Martowicz, A.; Uhl, T.; Silberschmidt, V.V. Modelling of non-linear crack-wave interactions for damage detection based on ultrasound-A review. J. Sound Vib. 2014, 333, 1097-1118. [CrossRef]

5. Burrascano, P.; Laureti, S.; Ricci, M. Harmonic Distortion Estimate for Damage Detection. In Proceedings of the NAECON 2018-IEEE National Aerospace and Electronics Conference, Dayton, OH, USA, 23-26 July 2018; pp. $274-279$.

6. Sutin, A.M.; Johnson, P.A. Non-linear elastic wave NDE II. Non-linear wave modulation spectroscopy and non-linear time reversed acoustics. In AIP Conference Proceedings; American Institute of Physics: College Park, MD, USA, 2005; Volume 760, pp. $385-392$.

7. Bruno, C.L.E.; Gliozzi, A.S.; Scalerandi, M.; Antonaci, P. Analysis of elastic non-linearity using the scaling subtraction method. Phys. Rev. B 2009, 79, 064108. [CrossRef]

8. Burrascano, P.; Di Bella, A.; Gliozzi, A.; Laureti, S.; Ricci, M.; Rizwan, M.K.; Tortello, M. A comparison of scaling subtraction and pulse compression methods for the analysis of elastic non-linearity. In Proceedings of Meetings on Acoustics ICU; American Institute of Physics: College Park, MD, USA, 2019; Volume 38, p. 065013.

9. Novak, A.; Bentahar, M.; Tournat, V.; El Guerjouma, R.; Simon, L. Non-linear acoustic characterization of micro-damaged materials through higher harmonic resonance analysis. Ndt E Int. 2012, 45, 1-8. [CrossRef]

10. Mechri, C.; Scalerandi, M.; Bentahar, M. Separation of damping and velocity strain dependencies using an ultrasonic monochromatic excitation. Phys. Rev. Appl. 2019, 11, 054050. [CrossRef]

11. Donskoy, D.; Sutin, A.; Ekimov, A. Non-linear acoustic interaction on contact interfaces and its use for non-destructive testing. Ndt E Int. 2001, 34, 231-238. [CrossRef]

12. Metya, A.K.; Tarafder, S.; Balasubramaniam, K. Non-linear lamb wave mixing for assessing localized deformation during creep. Ndt E Int. 2018, 98, 89-94. [CrossRef]

13. Sun, M.; Xiang, Y.; Deng, M.; Tang, B.; Zhu, W.; Xuan, F.Z. Experimental and numerical investigations of non-linear interaction of counter-propagating lamb waves. Appl. Phys. Lett. 2019, 114, 011902. [CrossRef]

14. Climent-Llorca, M.Á.; Miró-Oca, M.; Poveda-Martínez, P.; Ramis-Soriano, J. Use of higher-harmonic and intermodulation generation of ultrasonic waves to detecting cracks due to steel corrosion in reinforced cement mortar. Int. J. Concr. Struct. Mater. 2020, 14, 1-17. [CrossRef]

15. Chen, J.; Xu, Z.; Yu, Y.; Yao, Y. Experimental characterization of granite damage using non-linear ultrasonic techniques. Ndt $E$ Int. 2014, 67, 10-16. [CrossRef]

16. Sound System Equipment_Part 5: Loudspeakers; IEC 60268-5; International Electrotechnical Commission: Geneva, Switzerland, 2003.

17. Kay, S.M.; Marple, S.L. Spectrum analysis-A modern perspective. Proc. IEEE 1981, 69, 1380-1419. [CrossRef]

18. Daniell, P.J.; Volterra, V. The theory of functionals and of integral and integro-differential equations. Math. Gaz. 1932, 16, 59. [CrossRef]

19. Schetzen, M. The Volterra and Wiener Theories of Non-Linear Systems; Wiley: Hoboken, NJ, USA, 1980.

20. Schoukens, J.; Pintelon, R.; Dobrowiecki, T. Linear modeling in the presence of non-linear distortions. IEEE Trans. Instrum. Meas. 2002, 51, 786-792. [CrossRef]

21. Novak, A.; Simon, L.; Kadlec, F.; Lotton, P. Non-linear system identification using exponential swept-sine signal. IEEE Trans. Instrum. Meas. 2010, 59, 2220-2229. [CrossRef]

22. Rébillat, M.; Hajrya, R.; Mechbal, N. Non-linear structural damage detection based on cascade of Hammerstein models. Mech. Syst. Signal Process. 2014, 48, 247-259. [CrossRef]

23. Carpentieri, M.; Ricci, M.; Burrascano, P.; Torres, L.; Finocchio, G. Wideband microwave signal to trigger fast switching processes in magnetic tunnel junctions. J. Appl. Phys. 2012, 111, 07C909. [CrossRef]

24. Carpentieri, M.; Ricci, M.; Burrascano, P.; Torres, L.; Finocchio, G. Noise-like sequences to resonant excite the writing of a universal memory based on spin-transfer-torque MRAM. IEEE Trans. Magn. 2012, 48, 2407-2414. [CrossRef]

25. Cook, C. Pulse compression-key to more efficient radar transmission. Proc. IRE 1960, 48, 310-316. [CrossRef]

26. Laureti, S.; Colantonio, C.; Burrascano, P.; Melis, M.; Calabrò, G.; Malekmohammadi, H.; Sfarra, S.; Ricci, M.; Pelosi, C. Development of integrated innovative techniques for paintings examination: The case studies of The Resurrection of Christ attributed to Andrea Mantegna and the Crucifixion of Viterbo attributed to Michelangelo's workshop. J. Cult. Herit. 2019, 40, 1-16. [CrossRef]

27. Burrascano, P.; Ricci, M.; Battaglini, L.; Senni, L. Non-linear convolution and fourier series coefficients estimate. In Proceedings of the 2014 IEEE China Summit \& International Conference on Signal and Information Processing (ChinaSIP), Xi'an, China, 9-13 July 2014; pp. 728-732.

28. Burrascano, P.; Laureti, S.; Ricci, M.; Terenzi, A.; Cecchi, S.; Spinsante, S.; Piazza, F. A swept-sine pulse compression procedure for an effective measurement of intermodulation distortion. IEEE Trans. Instrum. Meas. 2019, 69, 1708-1719. [CrossRef]

29. Andreaus, U.; Baragatti, P. Cracked beam identification by numerically analysing the non-linear behaviour of the harmonically forced response. J. Sound Vib. 2011, 330, 721-742. [CrossRef] 